\title{
Growth and Decay Behaviour of a Sawtooth Profile in Non-Ideal Gas
}

\author{
R. Singh ${ }^{1}$ - L. P. Singh ${ }^{2}$
}

Published online: 24 April 2015

(C) Springer India Pvt. Ltd. 2015

\begin{abstract}
In the present paper, the progressive wave approach is used to analyze the main features of weakly nonlinear waves propagating in a non-ideal gas. An evolution equation, which characterizes the wave process in the high frequency domain and points out the possibility of wave breaking at a finite time, is derived. Further, we consider a sufficiently weak shock at the outset and study the propagation of the disturbance given in the form of a sawtooth profile. The effect of non-idealness on the formation of shock with planar and cylindrical symmetry is analyzed.
\end{abstract}

Keywords Non-ideal gas $\cdot$ Sawtooth profile $\cdot$ Progressive wave

\section{Introduction}

Discontinuity waves, also known as acceleration waves, are characterized by discontinuity in the normal first derivative of the flow variable rather than the variable itself. Therefore, for nonlinear systems, the analysis of these waves has been the subject of the great interest both from mathematical and physical point of view due to its application in a variety of fields such as astrophysics, nuclear science, geophysics, plasma physics and interstellar gas masses. In the study of a physical phenomenon ruled by a quasi-linear hyperbolic system of equations, it is theoretically possible to look for progressive wave solution. A general discussion of small-amplitude nonlinear progressive waves has been given by $[1,16]$ who considered a shockless solution of hyperbolic partial differential equations that depend on

$\triangle$ R. Singh

raghwendra.singh@gla.ac.in

L. P. Singh

lpsingh.apm@iitbhu.ac.in

1 Department of Mathematics, GLA University, Mathura 281 406, U.P., India

2 Department of Mathematical Sciences, Indian Institute of Technology, Banaras Hindu University, Varanasi 221005, India 
a single phase function. Using the perturbation method devised by [2-4] and [9] analyzed nonlinear wave propagation in different material media, while [5] presented a method for finding a small-amplitude high frequency wave solution of hyperbolic systems of quasilinear partial differential equations. When the gas flow takes place at a high temperature and density is too low the assumption that the gas is ideal is no longer valid. In recent years, several studies have been performed concerning the problem of weak shock waves in a non-ideal gas, in particular, by [6,7]. Recently [11] have studied evolutionary behaviour of acceleration waves in a perfectly conducting inviscid radiating gas permeated by a transverse magnetic field. Zhao et al. [15], has shown that the shock waves in a van der Waal's fluid exhibit a richer behaviour than that predicted by the ideal gas model, characterizing compressive shocks, rarefaction shocks, and shock splitting phenomena together with their admissibility; the physical meaning of van der Waals gas and its influence on wave motion may be seen in the papers [17-19]. Ambika et al. [20] have used the theory of progressive waves and some related procedures to study the attenuation and geometrical spreading of waves of finite and moderately small amplitudes influenced by the effects of non-linear convection in a non-ideal gas.

In the present study an asymptotic approach is used to analyze the growth and decay behaviour of a disturbance given in the form of a sawtooth profile that consists of a shock at the right and a sonic disturbance at the left in a non-ideal gas. The propagation of a sawtooth profile that ends in a tail shock can be treated in the same way.

\section{Governing Equations}

The basic equations for an unsteady one dimensional planar and non planar flow in a non-ideal gas, can be written as [12]

$$
\begin{gathered}
\rho_{t}+u \rho_{x}+\rho u_{x}+m \rho u / x=0, \\
u_{t}+u u_{x}+p_{x} / \rho=0, \\
p_{t}+u p_{x}+\rho a^{2}\left(u_{x}+m u / x\right)=0
\end{gathered}
$$

where $p$ is the pressure, $\rho$ is the gas density, $u$ is the velocity along the $x$-axis, $t$ the time, $x$ is the single spatial co-ordinate being either axial in flows with planar $(m=0)$ geometry or radial in cylindrically symmetric flows $(m=1)$ and $\gamma$ is the constant specific heat ratio. Here and throughout, non-numeric subscripts will denote partial differentiation with respect to the indicated variables unless stated otherwise. When the van der Waals excluded volume is sufficiently large, the gas is assumed to obey the van der Waals equation of state of the form [13]

$$
p(1-b \rho)=\rho R T, \quad e=c_{v} T=\frac{(1-b \rho) p}{(\gamma-1) \rho}, \quad S=c_{v} \ln \left[p(1-b \rho)^{\gamma} / \rho^{\gamma}\right]+\text { const },
$$

where $T$ is the temperature, $\mathrm{S}$ is the specific entropy, $R$ is the gas constant, and $c_{v}=R /(\gamma-1)$ is the specific heat at constant volume. It should be noted that this is a restricted form of the van der Waals equation for which the molecular attraction constant is zero. If the molecular attraction constant were nonzero, making the pressure non monotonic as a function of density, serious questions would arise as to whether the new mode propagates in a thermodynamically unstable state with negative compressibility. These questions are not addressed in this paper. Also, $a=(\gamma p / \rho(1-b \rho))^{1 / 2}$ is the sound speed. 
Equations (1)-(3) may be written in the matrix form as follows

$$
U_{t}^{i}+A^{i j} U_{x}^{j}+B^{i}=0, i, \quad j=1,2,3,
$$

where $U, B$ are column vectors and $A$ is the matrix of order $3 \times 3$, given as

$$
U=\left[\begin{array}{l}
\rho \\
u \\
p
\end{array}\right], \quad B=\left[\begin{array}{l}
m \rho u x^{-1} \\
0 \\
m \rho a^{2} u x^{-1}
\end{array}\right], \quad A=\left[\begin{array}{lll}
u & \rho & 0 \\
0 & u & \rho^{-1} \\
0 & \rho a^{2} & u
\end{array}\right] .
$$

Equation (5) is a system of quasilinear hyperbolic partial differential equations with eigenvalues $u+a, u-a$ and $u$ of the coefficient matrix $A$. The left and right eigenvectors of $A$ corresponding to the eigenvalue $u+a$ are

$$
\underset{\sim}{l}=[0, \rho a, 1], \quad r^{T}=\left[1, a / \rho, a^{2}\right]
$$

respectively. Here a superscript means transposition.

\section{Nonlinear Small Amplitude Progressive Waves}

We now look for an asymptotic solution of Eq. (5) which exhibits the features of progressive waves. Let us assume the following asymptotic expansion:

$$
U^{i}(x, t)=U_{0}^{i}+\varepsilon U_{1}^{i}(x, t, \xi)+O\left(\varepsilon^{2}\right),
$$

where $U_{0}^{i}$ is a known constant solution of (5) such that $B^{i}\left(U_{0}\right)=0$. The remaining terms in Eq. (7) are of a progressive wave nature. The choice of $\varepsilon$ depends on the physical problem to be studied. Let $\tau_{c h}$ be the characteristic time scale for the medium and $\tau_{a}$ be the attenuation time describing the dissipative mechanism; then $\varepsilon=\tau_{c h} / \tau_{a} \ll 1$. The variable $\xi$ is a 'fast variable' defined as $\xi=f(x, t) / \varepsilon$, where $f(x, t)$ is a phase function characterizing the wavefront.

It may be noted that the case $\varepsilon<<1$, which corresponds to the situation in which the characteristic frequency of the medium is much larger than the attenuation frequency, which characterises a high frequency propagation condition [8].

Introducing the Taylor's expansion of $A^{i j}$ and $B^{i}$ in the neighbourhood of the known constant solution $U_{0}^{i}$ and using (7), we have

$$
\begin{aligned}
A^{i j} & =A_{0}^{i j}+\varepsilon\left(\partial A^{i j} / \partial U^{k}\right)_{0} U_{1}^{k}+O\left(\varepsilon^{2}\right), \\
B^{i} & =\varepsilon\left(\partial B^{i} / \partial U^{k}\right)_{0} U_{1}^{k}+O\left(\varepsilon^{2}\right),
\end{aligned}
$$

where the subscript " 0 " means that the quantity involved is evaluated at the constant state $U_{0}$ at rest. Substituting Eqs. (7), (8) and (9) into (5), we obtain the following $O\left(\varepsilon^{0}\right)$ and $O\left(\varepsilon^{1}\right)$ equations respectively:

$$
\begin{gathered}
\left(A_{0}^{i j}-\lambda \delta_{j}^{i}\right) \partial U_{1}^{j} / \partial \xi=0, \\
\left(A_{0}^{i j}-\lambda \delta_{j}^{i}\right) \frac{\partial U_{2}^{j}}{\partial \xi}+\left(\frac{\partial U_{1}^{i}}{\partial t}+A_{0}^{i j} \frac{\partial U_{1}^{j}}{\partial x}\right) f_{x}^{-1}+U_{1}^{k}\left(\frac{\partial A^{i j}}{\partial U^{k}}\right)_{0} \frac{\partial U_{1}^{j}}{\partial \xi} \\
+f_{x}^{-1} U_{1}^{k}\left(\frac{\partial B^{i}}{\partial U^{k}}\right)_{0}=0,
\end{gathered}
$$


where $\lambda=-f_{t} / f_{x}$, and $\delta_{j}^{i}$ is the Kronecker delta. Equation (10) yields the characteristic polynomial

$$
\lambda^{2}\left(\lambda^{2}-a_{0}^{2}\right)=0,
$$

which yields non-zero eigenvalues $\pm a_{0}$ of $\underset{\sim}{A_{0}}$. Considering the velocity $\lambda=a_{0}$, the corresponding left and right eigenvectors of $A_{0}$ are given by Eq. (6) with subscript- -0 . Equation (10) shows that $\partial{\underset{\sim}{1}}_{1} / \partial \xi$ is collinear to $r_{0}$, and then ${\underset{\sim}{1}}_{1}$ may be written as

$$
{\underset{\sim}{1}}_{1}(x, t, \xi)=\alpha(x, t, \xi) \underset{\sim}{r_{0}}+\underset{\sim}{s}(x, t),
$$

representing a solution of Eq. (10). Here $\alpha(x, t, \xi)$ is the amplitude factor to be determined and $s^{i}$ (components of the column vector $\underset{\sim}{s}$ ) are integration constants, which are not of progressive wave nature and can therefore be taken as zero. The phase $f(x, t)$ is determined by

$$
f_{t}+a_{0} f_{x}=0
$$

and if $f(x, 0)=x-x_{0}$, then $f(x, t)=\left(x-x_{0}\right)-a_{0} t$.

Multiplying Eq. (11) by $l^{i}$, we obtain, along the characteristic curves associated with (13), the following evolution equation for $\alpha$ :

$$
\partial \alpha / \partial \tau+P_{0} \alpha \partial \alpha / \partial \xi+Q_{0} \alpha=0,
$$

where $\partial / \partial \tau=\partial / \partial t+a_{0} \partial / \partial x$ is the ray derivative taken along the rays and

$$
P_{0}=\left(\partial \lambda / \partial U^{k}\right)_{0} r_{0}^{k}=(\gamma+1) a_{0} /\left(2 \rho_{0}\right)>0, \quad Q_{0}=\left(l^{i} r^{i}\right)_{0}^{-1}\left(l^{j} r^{k} \partial B^{j} / \partial U^{k}\right)_{0}=m a_{0} / 2 x \text {. }
$$

Here, $Q_{0}^{-1}$ has the dimensions of time and may be taken as the attenuation time $\tau_{a}$ characterizing the medium. Equation (14) is a hyperbolic equation and its characteristic equations are

$$
\begin{gathered}
d \alpha / d \tau+m a_{0} \alpha / 2\left(x_{0}+a_{0} \tau\right)=0, \\
\frac{d \xi}{d \tau}=P_{0} \alpha .
\end{gathered}
$$

Equation (15) immediately gives

$$
\alpha(\xi, \tau)=\left[x_{0} /\left(x_{0}+a_{0} \tau\right)\right]^{m / 2} \phi\left(x_{0}, \xi_{0}\right),
$$

where $\phi\left(x_{0}, \xi_{0}\right)=\left.\alpha\right|_{\tau=0}$ with $\xi_{0}=\left.\varepsilon^{-1} f\right|_{\tau=0}$ and $x_{0}=\left.x\right|_{\tau=0}$.

The characteristic Eq. (16), which characterises plane $(m=0)$ and cylindrical $(m=1)$ waves, yields

$$
\xi= \begin{cases}\xi_{0}+\tau \mathrm{P}_{0} \phi\left(\mathrm{x}_{0}, \xi_{0}\right), & \text { for } \mathrm{m}=0, \\ \xi_{0}+\left\{2 \mathrm{P}_{0} \mathrm{x}_{0} \phi\left(\mathrm{x}_{0}, \xi_{0}\right) / \mathrm{a}_{0}\right\}\left\{\left(1+\mathrm{a}_{0} \tau / \mathrm{x}_{0}\right)^{1 / 2}-1\right\}, & \text { for } \mathrm{m}=1 .\end{cases}
$$

The existence of an envelope of the characteristics given by (17) gives evidence of the formation of a shock. It is evident that the shock is formed for $\tau>0$ only by those characteristics for which $(\partial \phi / \partial \xi)_{0}<0$, i.e., the shock formation will take place on the wave front if the initial phase of the pulse is compressive. The shock formation time for plane and cylindrical compressive waves turns out to be:

$$
\tau_{\mathrm{sh}}= \begin{cases}\min \left(\mathrm{P}_{0}\left|\partial \phi / \partial \xi_{0}\right|\right)^{-1}, & \text { for plane waves, } \\ \min \left[\left(\mathrm{x}_{0} / \mathrm{a}_{0}\right)\left(1+\mathrm{a}_{0} /\left(2 \mathrm{x}_{0} \mathrm{P}_{0}\left|\partial \phi / \partial \xi_{0}\right|\right)\right)^{2}-1\right], & \text { for cylindrical waves, }\end{cases}
$$

where the minimum is evaluated over an appropriate range of the quantities $x_{0}, \xi_{0}$. 


\section{Acceleration Waves}

The previous analysis may be used to study acceleration waves for the system (1-3). Let us assume that the acceleration front is described by the curve $f(x, t)=0$; across such a front the velocity is continuous but its first and higher order derivatives suffer finite jump discontinuity. In the neighbourhood of the front, the velocity $u$ may, therefore, be represented by an expansion

$$
u=\varepsilon u_{1}(x, t, \xi)+O\left(\varepsilon^{2}\right),
$$

where $u_{1}=0$ for $\xi<0$, and $u_{1}=O(\xi)$ for $\xi>0$. Now $u_{1}$ as an element of the column vector $U_{1}$ is given by (12), so we have [4]

$$
\alpha(x, t, \xi)= \begin{cases}0, & \text { if } \xi<0, \\ \xi \beta(x, t)+O\left(\xi^{2}\right), & \text { if } \xi>0,\end{cases}
$$

with $\beta=\left(\rho_{0} / a_{0}\right) \sigma$, where $\sigma=[\partial u / \partial x]$ denotes the jump in the velocity gradient across the acceleration front.

If we use (19) in (14), and then evaluate the resulting equation at the front $\xi=0$, we obtain

$$
\frac{d \sigma}{d t}+Q_{0} \sigma+\Lambda_{0} \sigma^{2}=0
$$

where $\Lambda_{0}=(\gamma+1) / 2$ and $Q_{0}=m a_{0} /(2 x)$, and the derivative $d / d t$ of any quantity, which is considered to be expressed on the front $f(x, t)=0$, is identical with the ordinary time derivative of the quantity.

Equation (20) is exactly the same as discussed in [10], and hence all the conclusions drawn therein follow at once. However, the solution of (20), which we shall be using in this section, can be written as follows:

$$
\sigma= \begin{cases}\sigma_{0} /\left(1+\sigma_{0} \Lambda_{0} \mathrm{t}\right), & \text { for } \mathrm{m}=0, \\ \sigma_{0}\left(1+\mathrm{a}_{0} \mathrm{t} / \mathrm{x}_{0}\right)^{-1 / 2} /\left[1+\left(2 \sigma_{0} \Lambda_{0} \mathrm{x}_{0} / \mathrm{a}_{0}\right)\left\{\left(1+\mathrm{a}_{0} \mathrm{t} / \mathrm{x}_{0}\right)^{1 / 2}-1\right\}\right], & \text { for } \mathrm{m}=1,\end{cases}
$$

where $\sigma_{0}$ is the value of $\sigma$ evaluated at $t=0$.

The above analysis shows that a compression pulse, however weak initially, always turns into a shock in a finite time. The state of motion in front of and behind the shock, designated respectively, by the subscripts ' 0 ' and ' 1 ', satisfy the following shock conditions [14].

$$
\rho_{1}=\rho_{0}(1+\delta), \quad u_{1}=\delta G /(1+\delta), \quad p_{1}=p_{0}+\delta \rho_{0} G^{2}(1+\delta)^{-1},
$$

where the shock strength parameter $\delta=\left(\rho_{1}-\rho_{0}\right) / \rho_{0}$ and the shock velocity $G$ are connected by

$$
G^{2}=\frac{2(1-\bar{b}) a_{0}^{2}(1+\delta)}{2-(\gamma-1) \delta-\bar{b} \delta(1+\delta)(\gamma-1)} .
$$

For a weak shock $\delta \ll 1$ Eq. (23) yields the first approximation as

$$
G=a_{0}\left(1+\Lambda_{0} \delta / 2\right),
$$

where $\Lambda_{0}$ is same as in Eq. (20).

Subsequently, Eq. (22) yield the first approximation

$$
\rho_{1}=\rho_{0}(1+\delta), \quad u_{1}=a_{0} \delta, \quad p_{1}=p_{0}(1+\gamma \delta),
$$

where $\delta$ is given by (23). 


\section{Decay of a Sawtooth Wave}

It is well known that shock waves after having travelled a large distance from the source become sufficiently weak so as to permit application of the weak shock relations (24) and (25). We therefore assume a sufficiently weak shock at the outset and study the propagation of the fluid velocity disturbance given in the form of a sawtooth profile. The left end of the profile located initially at $x_{0}$ travels with the sonic speed $a_{0}$ of the undisturbed fluid, whereas the shock at the right end located initially at $x_{s o}$ moves faster; $L_{0}$ is the initial length of the sawtooth profile. Suppressing the subscript -1 notation, let us denote by $u$ and $a$ the state of the rear side of the shock, which at any time $t$ is located at $x_{s}(t)=x_{0}+a_{0} t+L(t)$, where $L(t)$ is the length of the sawtooth at any time $t$. Then

$$
G=\frac{d x_{s}}{d t}=a_{0}+\frac{d L}{d t} .
$$

Also, on introducing (25) into (24), we get

$$
G=a_{0}+\frac{u \Lambda_{0}}{2}
$$

The sawtooth Profile, which runs into a medium at rest, furnishes, with constant $\partial u / \partial x$, the relation

$$
u=\sigma L(t),
$$

where $\sigma=(\partial u / \partial x)_{x-x_{0}=a_{0} t}$ is the slope of the profile at any time $t$, and is given by Eq. (21).

On using (28) into (27) and combining the resulting equation with (26), we obtain

$$
\frac{d L}{d t}=\frac{\sigma L \Lambda_{0}}{2} \text {. }
$$

Let $\sigma_{0}, L_{0}$ and $G_{0}$ be the values of $\sigma, L$ and $G$ respectively, at $t=0$. Then Eqs. (27) and (28), when evaluated at $t=0$ yield the following relation connecting $\sigma_{0}, L_{0}$ and $G_{0}$ :

$$
\sigma_{0}=\frac{2\left(G_{0}-a_{0}\right)}{L_{0} \Lambda_{0}}
$$

Equation (29), together with (21), yields on integration

$$
\frac{\mathrm{L}}{\mathrm{L}_{0}}= \begin{cases}\left(1+\sigma_{0} \Lambda_{0} \mathrm{t}\right)^{1 / 2}, & \text { for plane waves, } \\ \left\{1+\left(2 \sigma_{0} \Lambda_{0} \mathrm{x} / \mathrm{a}_{0}\right)\left[\left(1+\mathrm{a}_{0} \mathrm{t} / \mathrm{x}\right)^{1 / 2}-1\right]\right\}^{1 / 2}, & \text { for cylindrical waves, }\end{cases}
$$

where $\sigma_{0}$ is given by Eq. (30), and $\Lambda_{0}$ is same as in (20).

Using Eqs. (21) and (31) in (28), we obtain the decay behaviour of the sawtooth profile as follows:

$\mathrm{u}= \begin{cases}\mathrm{L}_{0} \sigma_{0}\left(1+\sigma_{0} \Lambda_{0} \mathrm{t}\right)^{-1 / 2}, & \text { for plane waves } \\ \mathrm{L}_{0} \sigma_{0}\left(1+\mathrm{a}_{0} \mathrm{t} / \mathrm{x}_{0}\right)^{-1 / 2}\left[\left(1-\mathrm{M}_{0}\right)+\mathrm{M}_{0}\left(1+\mathrm{a}_{0} \mathrm{t} / \mathrm{x}_{0}\right)^{1 / 2}\right]^{-1 / 2}, & \text { for cylindrical waves, }\end{cases}$

where $M_{0}=2 \sigma_{0} \Lambda_{0} x_{0} / a_{0}$.

\section{Result Discussion}

Equations (31) and (32) govern the variation in the length and velocity of the sawtooth wave with time respectively. The length $L / L_{0}$ and velocity $u / a_{0}$ are computed using (31) and (32) 


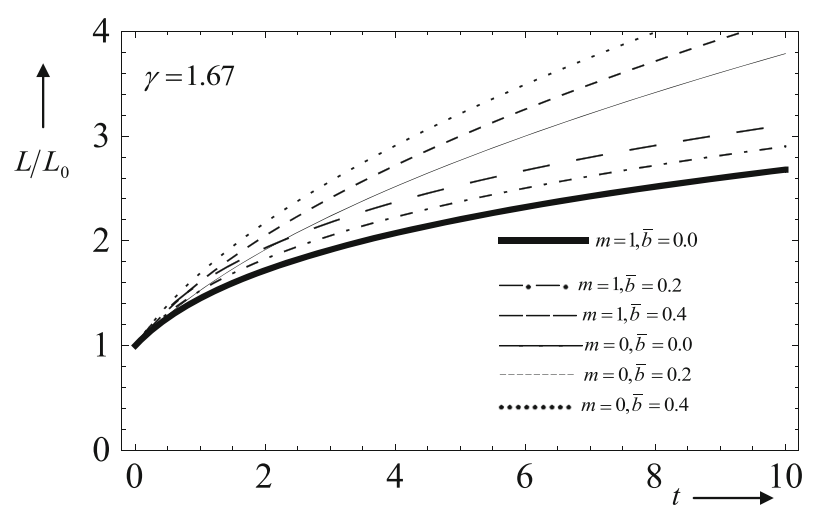

Fig. 1 Variation of length of sawtooth wave with time

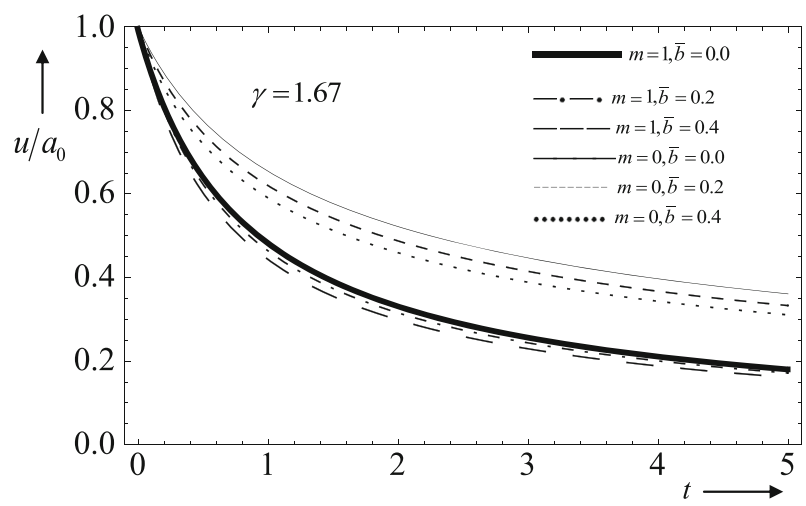

Fig. 2 Variation of velocity of sawtooth wave with time

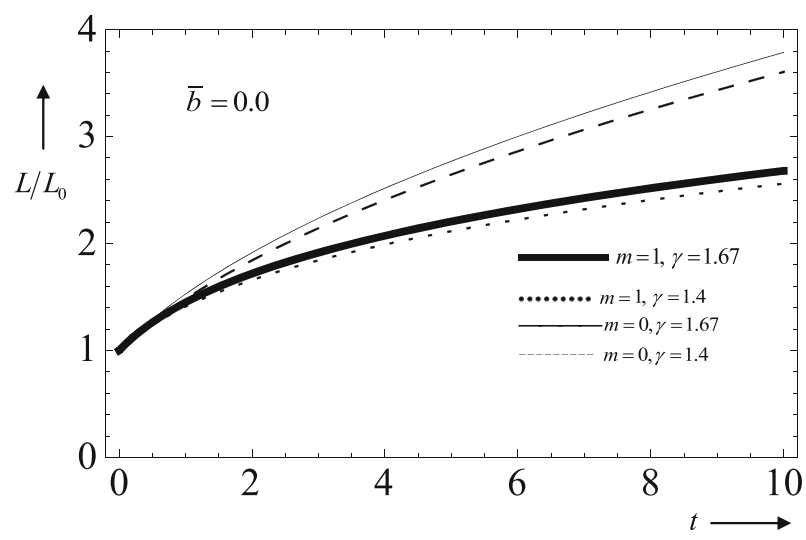

Fig. 3 Variation of length of sawtooth wave with time

for various values of parameter of non-idealness, $\bar{b}$ and specific heat ratio $\gamma$ for planar and cylindrically symmetric flows and presented in Figs. 1, 2, 3, 4, 5, 6.

The typical values of parameters involved in the computation are taken as:

$$
\begin{aligned}
\bar{b} & =0.0,0.2,0.4 . \\
\gamma & =1.4,1.67 .
\end{aligned}
$$




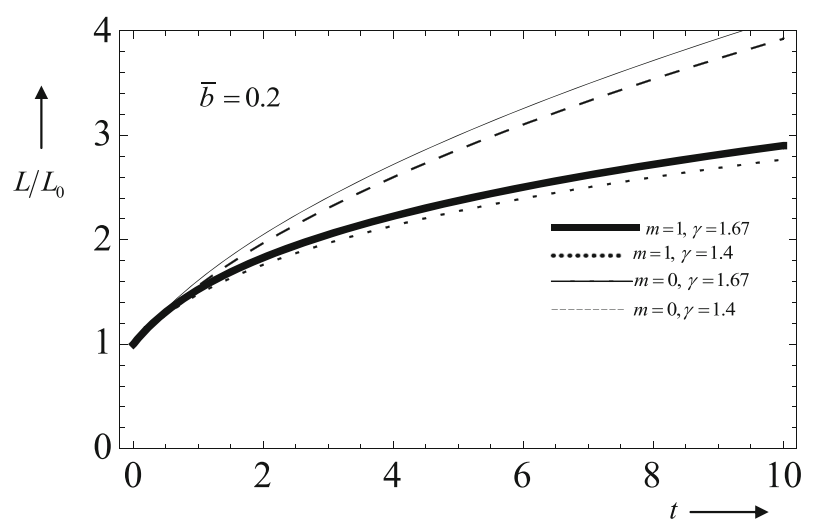

Fig. 4 Variation of length of sawtooth wave with time

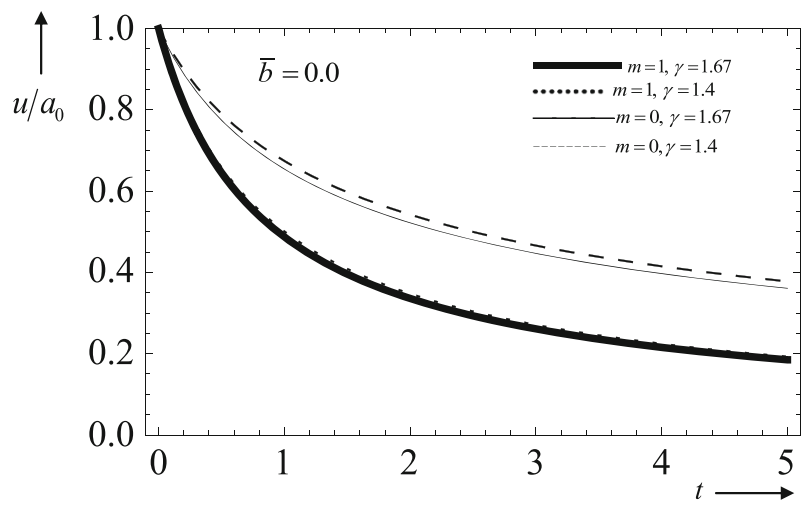

Fig. 5 Variation of velocity of sawtooth wave with time

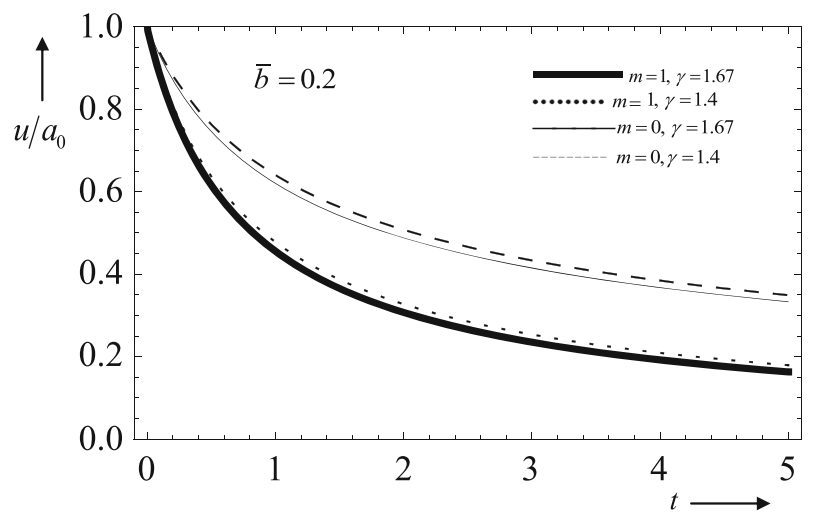

Fig. 6 Variation of velocity of sawtooth wave with time

For the case $\bar{b}=0.0$ which corresponds to ordinary gasdynamics, the Eqs. (31) and (32) reduce to the results outlined in [14].

From Figs. 1, 2, 3, 4, 5, 6 it is observed that the length $L / L_{0}$ increases with time, whereas the velocity decreases with time. Also the effect of increasing values of parameter of non- 
idealness $\bar{b}$ is to increase the length of profile whereas the same effect causes to decrease the velocity of the profile as evident from Figs. 1,2. This implies that the non-idealness of the gas will cause an early decay of the sawtooth wave as compared to ideal case. Also, the effect of non-idealness is more dominant in case of cylindrical symmetry as compared to plane case. It may be noted from Figs. 3, 4, 5, 6 that in both ideal and non-ideal gas the effect of increasing value of specific heat ratio $\gamma$ causes to increase (decrease) the length (velocity) of the profile.

\section{Conclusion}

The present paper uses the theory of progressive waves to analyze the main features of weakly nonlinear waves propagating in a non-ideal gas. An evolution equation, which characterizes the wave process in the high frequency domain and points out the possibility of wave breaking at a finite time, is derived. Further, we consider a sufficiently weak shock at the outset and study the propagation of the disturbance given in the form of a sawtooth profile. It is observed that the effect of non-idealness of the gas causes an early decay of the sawtooth wave as compared to ideal case. Also, the effect of non-idealness is more dominant in case of cylindrical symmetry as compared to plane case. Further, the effect of increasing values of specific heat ratio $\gamma$ is to increase (decrease) the length (velocity) of the profile.

Acknowledgments The first author acknowledges the financial support from the UGC, New Delhi, India, under the SRF scheme.

\section{References}

1. Choquet-Bruhat, Y.: Ondes asymptotiques et approchees pour des systemes d equations aux derivees partielles non lineaires. J. Math. Pures Appl. 48, 117-158 (1969)

2. Fusco, D., Engelbrecht, J.: The asymptotic analysis of nonlinear waves in rate dependent media. II Nuovo Cimento 80, 49-61 (1984)

3. Fusco, D.: Some comments on wave motions described by non homogeneous quasilinear first order hyperbolic systems. Meccanica 17, 128-137 (1982)

4. Germain, P.: Progressive waves, in: Jahrbuch der DGLR, pp. 11-30 (1971)

5. Hunter, J.K., Keller, J.B.: Weakly non-linear heigh frequency wave. Commun. Pure Appl. Math. 36, 547-569 (1983)

6. Madhumita, G., Sharma, V.D.: Imploding cylindrical and spherical shock waves in a non-ideal medium. J. Hyperbolic Differ. Equ. 1, 521-530 (2004)

7. Pandey, M., Sharma, V.D.: Interaction of a characteristic shock with a weak discontinuity in a non-ideal gas. Wave Motion 44, 346-354 (2007)

8. Seymour, B.R., Varley, E.: High frequency periodic disturbances in dissipative systems. I. Small amplitude finite rate theory. Proc. R. Soc. Lond. A 314, 387-415 (1970)

9. Sharma, V.D., Singh, L.P., Ram, R.: The progressive wave approach analyzing the decay of a sawtooth profile in magnetogasdynamics. Phys. Fluids 30, 1572-1574 (1987)

10. Sharma, V.D., Menon, V.V.: Further comments on the behaviour of acceleration waves of arbitrary shape. J. Math. Phys. 22, 683-684 (1981)

11. Singh, L.P., Singh, R., Ram, S.D.: Evolution and decay of acceleration waves in perfectly conducting inviscid radiative magnetogasdynamics. Astrophys. Space Sci. 342, 371-376 (2012)

12. Wu, C.C., Roberts, P.H.: Structure and stability of a spherical shock wave in a Van der Waals gas. Quart. J. Mech. Appl. Math. 49, 501-543 (1996)

13. Roberts, P.H., Wu, C.C.: Structure and stability of a spherical implosion. Phys. Lett. A 213, 59-64 (1996)

14. Zirep, J.: Theoretical Gasdynamics. Springer, Berlin (1978)

15. Zhao, N., Mentrelli, A., Ruggeri, T., Sugiyama, M.: Admissible shock waves and shock-induced phase transitions in a van der Waals fluid. Phys. Fluids 23, 086101 (2011) 
16. Boillat, G.: Ondes asymptotiques nonlin'eaires. Ann. Mat. Pura Appl, IV 91, 31-44 (1976)

17. Thompson, P.A.: A fundamental derivative in gas dynamics. Phys. Fluids 14, 1843-1849 (1971)

18. Cramer, M.S., Sen, R.: Exact solutions for sonic shocks in van der Waals gases. Phys. Fluids 30, 377-385 (1987)

19. Menikoff, R., Plohr, B.J.: The Riemann problem for fluid flow of real materials. Rev. Mod. Phys. 61, 75-130 (1989)

20. Ambika, K., Radha, R., Sharma, V.D.: Progressive waves in non-ideal gases. Int. J. Nonlinear Mech. 67, 285-290 (2014) 